\title{
Comparative Study of Human and Automated Screening for Antinuclear Antibodies by Immunofluorescence on HEp-2 Cells
}

\author{
Yousr Gorgi ${ }^{1,2,}$, Tarak Dhaouadi $^{1}$, Imen Sfar ${ }^{1,2}$, Youssra Haouami ${ }^{1,2}$, Taieb Ben Abdallah ${ }^{1}$, \\ Giuseppe Raso ${ }^{3}$, Donato Cascio ${ }^{3}$, Marco Cipolla ${ }^{3}$, Vincenzo Taormina ${ }^{3}$, Alessandro Fauci ${ }^{3}$, \\ Ignazio Brusca ${ }^{4}$, Giuseppe Friscia ${ }^{5}$, Amel Benammar Elgaaïed ${ }^{6}$, Raja Marrakchi Triki ${ }^{6}$, \\ Asma Gati ${ }^{6}$, Melika Ben Ahmed ${ }^{7}$ and Hechmi Louzir ${ }^{7}$
}

${ }^{1}$ Laboratory of Research in Immunology of Renal Transplantation and Immunopathology (LR03SP01), Charles Nicolle Hospital, University Tunis El Manar, Tunisia

${ }^{2}$ Laboratory of Immunology, Charles Nicolle Hospital, Tunis, Tunisia

${ }^{3}$ Dipartimento di Fisica e Chimica, Università Degli Studi di Palermo, Palermo, Italy

${ }^{4}$ Ospedale Buccheri La Ferla Fatebenefratelli, Palermo, Italy

${ }^{5}$ Ospedale di Sciacca, Agrigento, Italy

${ }^{6}$ Faculty of Sciences, University Tunis El Manar, Tunisia

${ }^{7}$ Laboratory of Immunology, Pasteur Institute Tunis, Tunisia

\begin{abstract}
Background: Several automated systems had been developed in order to reduce inter-observer variability in indirect immunofluorescence (IIF) interpretation. We aimed to evaluate the performance of a processing system in antinuclear antibodies (ANA) screening on HEp-2 cells.

Patients and Methods: This study included 64 ANA-positive sera and 107 ANA-negative sera that underwent IIF on two commercial kits of HEp-2 cells (BioSystems ${ }^{\circledR}$ and Euroimmun ${ }^{\circledR}$ ). IIF results were compared with a novel automated interpretation system, the "CyclopusCADImmuno®" (CAD).

Results: All ANA-positive sera images were recognized as positive by CAD (sensitivity $=100 \%$ ), while $17(15.9 \%)$ of the ANA-negative sera images were interpreted as positive (specificity $=84.1 \%), \mathrm{K}=0.799(\mathrm{SD}=0.045)$. Comparison of IIF pattern determination between human and CAD system revealed on HEp-2 (BioSystems ${ }^{\circledR}$ ), a complete concordance in $6(9.37 \%)$ sera, a partial concordance (sharing of at least 1 pattern) in $42(65.6 \%)$ cases and in $16(25 \%)$ sera the pattern interpretation was discordant. Similarly, on HEp-2 (Euroimmun $\left.{ }^{\circledR}\right)$ the concordance in pattern interpretation was total in $5(7.8 \%)$ sera, partial in $39(60.9 \%)$ and absent in $20(31.25 \%)$. For both tested HEp-2 cells kits agreement was enhanced for the most common patterns, homogenous, fine speckled and coarse speckled. While there was an issue in identification of nucleolar, dots and nuclear membranous patterns by CAD.

Conclusion: Assessment of ANA by IIF on HEp-2 cells using the automated interpretation system, the "CyclopusCADImmuno $®$ " is a reliable method for positive/negative differentiation. Continuous integration of IIF images would improve the pattern identification by the CAD.
\end{abstract}

Keywords: Autoantibodies, HEp-2, Immunofluorescence, autoimmune diseases, automated screening, standardization.

\section{INTRODUCTION}

Antinuclear antibodies (ANA) are clinically useful markers of several chronic autoimmune diseases called connectivitis. Indirect immunofluorescence (IIF) which was described in 1958 has become the standard method for the detection of several autoantibodies including ANA [1]. In spite of the development of several assays like ELISA or microarrays, IIF on HEp-2 cells remains the "gold standard" for ANA screening [2]. Currently, there is no consensus on the potential

*Address correspondence to this author at the Laboratory of Research in Immunology of Renal Transplantation and Immunopathology (LR03SP01), University Tunis El Manar, Charles Nicolle Hospital, Bd 9 Avril, 1006 Bab Saadoun, Tunis, Tunisia; Tel: +21698341791; Fax: +216 71561156;

E-mail: gorgi.yousr@gmail.com factors that could influence IIF results (culture condition, fixative procedure of HEp-2 cells, starting dilution, antiglobuline specificity, etc.). In practice, most laboratories perform ANA screening at the $1 / 80^{\text {th }}$ dilution or less frequently at $1 / 160^{\text {th }}$. It is worth to note that 10 to $15 \%$ of healthy adults has positive ANA at the $1 / 80^{\text {th }}$ titer and $5 \%$ at $1 / 160^{\text {th }}$, and this frequency tends to increase with aging mostly in women after 60 years old. Obviously, positivity at these titers has no clinical significance.

The type and the localization of the fluorescence define the IIF pattern. The terminology of IIF images is not consensual, however the revised nomenclature made within the CANTOR project [1] would provide homogeneity in image definitions. This revised 
taxonomy outlines 6 groups of patterns: 1) Membranous nuclear patterns, 2) Nucleoplasmic patterns, 3) nucleolar patterns, 4) spindle apparatus patterns, 5) Cytoplasmic patterns and 6) negative [1].

Besides, inter-laboratories and intra-laboratory discrepancies in terms of negative or positive aspects and in terms of pattern recognition depends on:

- $\quad$ The variability inherent to the used kit of HEp-2 cells

- $\quad$ The reading system: microscope sensitivity, and the kind of the used lamp (HBO or LED)

- The threshold of positivity and the pattern identification, in fact usually several patterns overlap. Therefore, the skill of the observer is a key point.

Since the increase of ANA test requests, which led to speed up the reading time on microscope, the lack of standardization and inter-observer variability, several automated systems of image reading had been developed. These systems would be used in second reading after that of the human expert or within the framework of apprenticeship of the inexperienced juniors. Various stages are necessary: 1) the acquisition automated by the image, 2) a quality control of the image, 3 ) the segmentation of the image for the detection of the object, 4) the extraction of the characteristics to describe the object and the classification of the detected objects. The acquisition of a quality image depends on the automated tuning, on the adjustment of the intensity of the image, on the quality control of the sharpness and on the luminosity, on the detection of artifacts, on the real time evaluation of the aspect of fluorescence and on the calibration of the fluorescence. The advantage is to obtain comparable results between laboratories, which require a standardization of the IIF with a measure of the intensity in units of fluorescence and a calibrated system allowing in particular comparable studies of groups in various laboratories. The system has to allow, thanks to adapted software, the memorization of the acquired images and their archiving for possible comparisons.

Different systems are in development and, to our knowledges, those currently proposed for routine analysis with image interpretation are [3]:

- $\quad$ Aklides System (Medipan, Berlin, Germany) on HEp-2: negative / positive discrimination, 5 patterns
- Nova View (Werfen, INOVA Diagnostics Inc., Barcelona, Spain) on HEp-2: negative / positive discrimination, 6 patterns

- Zenit G-Sight A (Menarini Diagnostics, Florence, Italy) on HEp-2 or HEp-2000: negative / positive discrimination, 5 patterns

- $\quad$ EuroPattern (Euroimmun, Lübeck, Germany) on HEp-2 negative / positive discrimination, 5 patterns

- Helios (Aesku Diagnostics, Wendelsheim, Germany) on HEp-2: negative / positive discrimination

- Image Navigator (Immuno Concepts, Sacramento, United States) on HEp-2: negative / positive discrimination.

The present study was undertaken to evaluate the performances of a new system, the CyclopusCADImmuno ${ }^{\circledR}$, which is still undergoing a process of development and education.

\section{MATERIAL AND METHODS}

\section{Patients and Controls}

In this study, 64 ANA-positive sera from patients with a confirmed diagnosis of systemic lupus erythematosus (SLE) and 107 ANA-negative sera from healthy blood donors were included. The antigentargets of the collected ANA-positive sera were previously determined by both IIF on CrithidiaLucilae for the anti-dsDNA antibodies and immunodot (Innolia $\AA$ ) for the antibodies to extractable nuclear antigens (ENA). Sixty-three $(98.4 \%)$ of the ANApositive sera contained at least 2 or more target specificities. ANA titers were considered high when it surpassed the $1 / 320^{\text {th }}$ dilution. Characteristics of the ANA-positive sera are recorded in Table 1.

All patients and controls gave written informed consent to participate in the study, and patient anonymity was preserved using documents and methods approved by the local Ethics committee of Charles Nicolle Hospital.

\section{Methods}

\section{Indirect Immunofluorescence (IIF) Assay}

ANA detection was performed by IIF using 2 commercial kits of HEp-2 cells: BioSystems ${ }^{\circledR}$ and 
Euroimmun ${ }^{\circledR}$. The complete incubation process was carried out manually: 1) first, the sera diluted at $1 / 80^{\text {th }}$ were incubated with HEp-2 cells for 30 minutes at room temperature, 2) Second, 3 washing of 5 minutes each with PBS-Tween, 3) third, incubation with goat antihuman $\lg G$ conjugated with fluorescein isothiocyanate and 4) fourth, after 3 washing, slides were embedded with a mounting medium. The ANA-negative sera were tested only in Euroimmun® HEp-2 kit.

\section{Human Evaluation of ANA}

Two skilled human experts whose experience in IIF analysis is about 18 and 9 years, respectively, evaluated the HEp-2 slides. Slides were interpreted within the Motic $₫$ BA310 Epi-LED FL microscope. The two experts worked blindly and independently without reference to each other or to the evaluated software's readings. The reported patterns were: 1 ) homogenous, 2) Speckled (fine speckled and/or coarse speckled), 3) nuclear dots, 4) nucleolar, 5) membranous nuclear, and 6) negative.

\section{Automated Evaluation by the} CyclopusCADImmuno ${ }^{\circledR}$

The system used in this study is the CyclopusCADImmuno $((C A D)$, powered by CyclopusCADsrl, a spin-off of University of Palermo. It consists of an informatics program for image analysis using artificial intelligence. The CAD is connected to a high-resolution camera (AESKU® LED 3002), which is itself linked to the same fluorescence microscope used by human experts. The CAD system and the highresolution camera were acquired through the AIDA project funded under the European Cooperation ENPI Italy-Tunisia.

\section{Statistical Analysis}

The agreement between human and CAD pattern recognition was evaluated using $\mathrm{k}$ statistics (SPSS 11 Inc. Chicago, Illinois, USA). Based on $\mathrm{k}$ test, the agreement is poor when it ranges between 0 and 0.2 , fair between 0.2 and 0.4 , moderate between 0.4 and 0.6 , substantial between 0.6 and 0.8 , and almost perfect between 0.8 and 1 . When $\mathrm{k}$ is $<0$ the tests' results are considered contradictory. Analysis of the influence of ANA titers on discrepancies between human and $C A D$ interpretations was performed using chi-square test or fisher's exact test for small numbers (SPSS 11 Inc. Chicago, Illinois, USA). Values of $p<$ 0.05 were considered to be statistically significant.

\section{RESULTS}

In total, 64 ANA-positive sera and 107 ANAnegative sera were included in this study. Fifty $(78.12 \%)$ sera had elevated titer of ANA and 63 $(98.4 \%)$ had at least two or more target-antigen specificities (Table 1).

Table1: Characteristics of the 64 ANA-Positive Sera

\begin{tabular}{|c|c|}
\hline ANA-positive sera & $\mathbf{N}=\mathbf{6 4}$ \\
\hline \hline Elevated titer $\geq 1 / 320^{\text {th }}$ & $50(78.12 \%)$ \\
\hline Mixed pattern (human interpretation) & $34(53.1 \%)$ \\
\hline Two or more specificities of ANA & $63(98.4 \%)$ \\
\hline Anti-dsDNA antibodies & $37(57.8 \%)$ \\
\hline Anti-Sm antibodies & $22(34.37 \%)$ \\
\hline Anti-histone antibodies & $28(43.75 \%)$ \\
\hline Anti-ribosome antibodies & $10(15.6 \%)$ \\
\hline Anti-RNP antibodies & $16(25 \%)$ \\
\hline Anti-SSA/Ro52 & $30(46.87 \%)$ \\
\hline Anti-SSA/Ro60 & $22(34.37 \%)$ \\
\hline Anti-SSB/La & $22(34.37 \%)$ \\
\hline
\end{tabular}

Comparison between BioSystems and Euroimmun Patterns' Interpretation by Human Experts

Agreement between the two human experts was total $(100 \%)$. Globally $23(35.9 \%)$ sera were in a total agreement for the IIF patterns detected on the two tested HEp-2 kits. A partial agreement (sharing of one IIF pattern) was found in $28(43.75 \%)$ cases while a complete disparity was observed in $13(20.3 \%)$ sera. Besides, agreement was moderate for the most common IIF patterns, homogenous, speckled, nuclear dots and membranous nuclear; $\mathrm{k}=0.43, \mathrm{k}=0.56$, $\mathrm{K}=0.488$ and $\mathrm{K}=0.435$ respectively. Agreement between the two tested HEp-2 kits was not correlated to the titer of ANA.

\section{Comparison between BioSystems and Euroimmun Patterns' Interpretation by CyclopusCAD}

IIF patterns' identification was totally concordant in $17(26.6 \%)$ sera, partly agreeing in $31(48.5 \%)$ cases and completely disagreeing in only $16(25 \%)$ samples. Agreement was poor for the homogenous and nucleolar patterns ( $\mathrm{k}=0.143$ and $\mathrm{k}=0.124)$, fair for the nuclear dot pattern $(\mathrm{k}=0.333)$ and moderate for the speckled pattern $(k=0.421)$. Again, the agreement between the generated patterns of the two kits was not associated to ANA titer. 
Performance of the CyclopusCAD in Negative/Positive Discrimination

All ANA-positive sera of the lupus patients were recognized as positive, therefore its sensitivity is about $100 \%$. By contrast, 17 (15.9\%) of the ANA-negative sera were recognized as positive by the CyclopusCAD system, hence its specificity is about $84.1 \%$ (Table 2). The concordance between human expert and software interpretations was $90.05 \%$ and agreement was almost perfect, $k=0.8$.It is worth to note that all of the 17 ANAnegative sera, which were identified by the CyclopusCAD as positive, had a cytoplasmic pattern.

Table 2: Negative/Positive Discrimination of the CyclopusCAD

\begin{tabular}{|c|c|c|}
\hline CyclopusCADImmuno & Positive & Negative \\
\hline \hline Lupus sera (ANA-positive) & 64 & 0 \\
\hline Control sera (ANA-negative) & 17 & 90 \\
\hline K value & \multicolumn{2}{|c|}{0.8} \\
\hline Sensitivity & \multicolumn{2}{|c|}{$100 \%$} \\
\hline Specificity & \multicolumn{2}{|c|}{$84.1 \%$} \\
\hline Positive predictive value & \multicolumn{2}{|c|}{$79 \%$} \\
\hline Negative predictive value & \multicolumn{2}{|c|}{$100 \%$} \\
\hline
\end{tabular}

Performance of the CyclopusCAD in Identification of IIF Patterns on HEp-2 BioSystems ${ }^{\circledR}$

Using human experts as reference, the CyclopusCAD identified the rightlIF pattern in 6 $(9.37 \%)$ sera, while in $42(65.6 \%)$ cases it recognized at least one of the mixed patterns. Inversely, in 16 (25\%) ANA-positive sera, the CAD did not pinpoint the correct IIF pattern. Comprehensive analysis of the patterns showed (Table 3):
1) Moderate agreements for homogenous, speckled, and coarse speckled patterns

2) Incongruity for nuclear dots, nucleolar, membranous nuclear patterns

Besides, discrepancies in IIF pattern identification were encountered more frequently in sera with low titers of ANA but differences failed to reach the threshold of significance.

\section{Performance of the CyclopusCAD in Identification of IIF Patterns on HEp-2 Euroimmun ${ }^{\circledR}$}

The CyclopusCAD recognized the correct IIF pattern in five $(7.8 \%)$ cases, whereas it pinpointed one of the patterns in $39(60.9 \%)$ sera. However, the CAD system failed to determine the exact pattern in 20 $(31.25 \%)$ cases. Detailed examination revealed (Table 4):

1) Moderate agreement for the coarse speckled pattern

2) Fair agreement for homogenous, speckled and fine speckled patterns

3) Poor agreement for nuclear dots, nucleolar and membranous nuclear patterns

Furthermore, agreement was non-significantly better in case of high titers of ANA.

\section{DISCUSSION}

In the present study, we enrolled 64 ANA-positive sera from lupus-diagnosed patients and 107 ANAnegative sera from healthy blood donors. The ANApositive sera were tested with both BioSystems ${ }^{\circledR}$ and Euroimmun ${ }^{\circledR} \mathrm{HEp}-2$ kits, while ANA-negative sera with

Table 3: Agreements between Human Experts and the CyclopusCAD on HEp-2 BioSystems ${ }^{\circledR}$

\begin{tabular}{|c|c|c|c|c|}
\hline Pattern & $\begin{array}{c}\text { Concordant } \\
(+/-)\end{array}$ & $\begin{array}{c}\text { Discordant } \\
\text { Hum+/CAD- }\end{array}$ & $\begin{array}{c}\text { Discordant } \\
\text { Hum-/CAD+ }\end{array}$ & 16 \\
\hline \hline Homogenous & $47(17 / 30)$ & 1 & 7 & 0.476 \\
\hline Speckled & $51(39 / 12)$ & 6 & 12 & 0.506 \\
\hline Fine speckled & $42(17 / 25)$ & 10 & 13 & 0.302 \\
\hline Coarse speckled & $51(14 / 37)$ & 4 & 13 & -0.082 \\
\hline Nuclear dots & $48(0 / 48)$ & 3 & 3 & 0.013 \\
\hline Nucleolar & $45(2 / 43)$ & 6 & 0.034 \\
\hline
\end{tabular}

+: present, -: absent, Hum: human expert interpretation. 
Table 4: Agreements between Human Experts and the CyclopusCAD on HEp-2 Euroimmun®

\begin{tabular}{|c|c|c|c|c|}
\hline Pattern & $\begin{array}{c}\text { Concordants } \\
(+/-)\end{array}$ & $\begin{array}{c}\text { Discordants } \\
\text { Hum+/CAD- }\end{array}$ & $\begin{array}{c}\text { Discordants } \\
\text { Hum-/CAD+ }\end{array}$ & 23 \\
\hline \hline Homogenous & $39(25 / 14)$ & 2 & 13 & 0.275 \\
\hline Speckled & $42(29 / 13)$ & 9 & 15 & 2 \\
\hline Fine speckled & $43(11 / 32)$ & 6 & 16 & 0.27 \\
\hline Coarse speckled & $58(3 / 55)$ & 4 & 4 & -0.03 \\
\hline Nuclear dots & $47(0 / 47)$ & 1 & -0.043 \\
\hline Nucleolar & $58(0 / 58)$ & 2 & -0.031 \\
\hline
\end{tabular}

+: present, -: absent, Hum: human expert interpretation.

Euroimmun ${ }^{\circledR}$ only. All images were interpreted independently by two human experts and by the system undergoing evaluation, the CyclopusCADImmuno®.

Comparative evaluation of the IIF patterns by human experts of the two tested HEp-2 kits revealed a concordance rate of $79.7 \% \quad(35.9 \%$ complete agreement and $43.75 \%$ partial agreement). The best agreements involved specifically the most common IIF patterns, homogenous, speckled, nuclear dots and membranous nuclear. This results corroborates that the study of Copple et al. [4], in which 5 HEp-2 kits (Kallestad $\AA$, ImmunoConcepts $\AA$, Zeus $₫$, Euroimmun $\AA$ and Inova ${ }^{\circledR}$ ) were compared using 372 sera (50 SLE, 45 rheumatoid arthritis, 35 scleroderma, 20 Sjögren syndrome, 10 polymyositis, 12 reference sera from the CDC, 100 healthy blood donors and 100 sera from private laboratories). In this study, three skilled technologists made evaluation independently and blindly to sample classification and each other's reading [4]. Concordance between the 3 readers varied from $96 \%$ to $99 \%$ [4]. Overall, the percentage of agreement for the $5 \mathrm{HEp}-2$ assays was $78 \%$ and varied from $44 \%$ in the scleroderma sera to $93 \%$ in the healthy blood donor group [4]. Furthermore, the authors reported discrepancies in titers' estimation that reached for some specimens from to $1 / 80^{\text {th }}$ to $1 / 1280^{\text {th }}$ [4]. It has been proposed that the use of a polyconjugate versus an IgG-specific conjugate results in variability among the 5 tested kits. In fact, polyconjugate ( $G, A$ and $M$ ) or total IgG (heavy and light chain) leads to the detection of the lgM class of ANA associated with rheumatoid arthritis, medications, and ageing, which are usually clinically insignificant. Besides, other factors such as culture conditions, fixation procedures, $\mathrm{pH}$, assay temperature and many others may cause variability in both generation IIF pattern and ANA titers [5].

Evaluation of the CyclopusCAD performance in negative/positive discrimination revealed comparable characteristics with commercialized systems (Table 5) [3]. Besides, in the present study the 17 sera that were falsely considered as ANA-positive had a cytoplasmic pattern. In fact, such cytoplasmic fluorescence is able to be considered as a nucleoplasmic pattern if the segmentation of the nuclei is not perfect. In this regard, Tonti et al. [6] proposed a novel approach to the segmentation of HEp-2 cells relying on:

1) image acquisition

2) image enhancement

Table 5: Comparative Analysis of the CyclopusCAD with Commercialized Systems [3]

\begin{tabular}{|c|c|c|c|c|c|c|c|}
\hline System & Aklides & EuroPattern & Helios & Image Navigator & NovaView & G-Sight & CyclopusCAD \\
\hline \hline Positive & $90 / 92$ & $89 / 92$ & $90 / 92$ & $88 / 92$ & $86 / 92$ & $91 / 92$ & $64 / 64$ \\
\hline Negative & $30 / 34$ & $29 / 34$ & $32 / 34$ & $32 / 34$ & $32 / 34$ & $27 / 34$ & $90 / 107$ \\
\hline Sensitivity & 97.8 & 96.7 & 97.8 & 95.7 & 93.5 & 98.9 & 100 \\
\hline Specificity & 88.2 & 85.3 & 94.1 & 94.1 & 94.1 & 79.4 & 84.1 \\
\hline PPV & 95.7 & 94.6 & 97.8 & 97.7 & 97.7 & 92.8 & 79 \\
\hline NPV & 93.7 & 90.6 & 94.1 & 88.8 & 84.2 & 96.4 & 100 \\
\hline
\end{tabular}

PPV: positive predictive value, NPV: negative predictive value. 
3) image classification as with smooth or rough texture

4) Image normalization

5) Marker extraction: identification of foreground regions and then separation of clustered cells

6) Final and critical segmentation of the nuclei

Otherwise, comparative analysis of IIF patterns' interpretation between human experts and the CyclopusCAD revealed a good global concordance with either BioSystems $®$ orEuroimmun $®, 75 \%$ and $68.75 \%$ respectively. These overall performances are close to that of EuroPattern ${ }^{\circledR}$ system, which was estimated at $79 \%$ [3]. While, it may surpass those of the three other systems, Aklides ${ }^{\circledR}(52 \%)$, NovaView $\circledR$ $(54 \%)$ and G-Sight $\circledast(63 \%)$ [3]. It is worth to note that in the comparative study of these commercialized systems used only single pattern sera [3]. In another study, including sera with mixed pattern, the global performance of the EuroPattern $®$ system was estimated at $48.9 \%$ [7]. Therefore, recognizing mixed patterns is critical. Moreover, it has been reported in Aklides system [8, 9], that distinction of patterns became problematic when there were 2 or 3 ANA specificities. Hence, considering the fact $98.4 \%$ of tested sera in our study had 2 or more specificities, the capacity of the CyclopusCAD, a system which is still ongoing licensing, is promising.

To improve performance of automated systems, including the CyclopusCAD, a greater number of images must be acquired using 2 or 3 dilutions in order to ease interpretation of mixed patterns. It is of note, that aside from G-sight system which was developed with HEp-2 and HEp-2000 (transfected with SSA antigen), all other systems are to be considered as closed. In fact, the automated systems recognize patterns only on the HEp-2 kit with which it had been licensed. Consequently, if reproducibility between laboratories using the same system would be satisfying, a certain variability will persist between those using different systems. Therefore, commercialized and ongoing development systems need to be educated on a wide range of different HEp2 kits.

Besides, the big laboratories with a high debit of ANA requests tend to have a diagnostic approach in 2 stages. A first stage of positive/negative screening then a second stage of titration via serial dilutions. A calibration of the automated system according to the intensity of the fluorescence would allow obtaining straightaway and exactly the title of the AAN in a single stage. Eventually, the CyclopusCAD system will be associated with an automaton allowing the preparation of HEp-2 and connected directly with a microscope allowing a fast acquisition and an interpretation of the images and their archiving without any human interference.

\section{CONCLUSION}

Assessment of ANA by IIF on HEp-2 cells using the automated interpretation system, the "CyclopusCADImmuno $®$ " is a reliable method for positive/negative differentiation. Continuous integration of IIF images would improve the pattern identification by the CAD.

\section{ACKNOWLEDGEMENTS}

This work was granted and supported by the Laboratory of Research in Immunology of Renal Transplantation and Immunopathology (LR03SP01). University Tunis El Manar. Charles Nicolle Hospital. Tunis, TUNISIA

\section{DISCLAIMER}

This document was produced with the financial assistance of the European Union in the frame of the ENPI CT Italy-Tunisia 2007-2013. The views expressed herein can in no way be taken to reflect the official opinion of the European Union. Sole responsibility for the views, interpretations or conclusions contained in this document lies with the authors. Neither the European Commission nor Management Structures of the Program can be held responsible for the accuracy, completeness or use that may be made of the information contained herein.

\section{COMPETING INTERESTS}

No benefits in any form have been received or will be received from a commercial party related directly or indirectly to the subject of this article.

\section{AUTHORS' CONTRIBUTION}

Pr. Gorgi Yousr proposed the study. Tarak Dhaouadi and Imen Sfar analyzed the data. All authors contributed to the design and interpretation of the study. Pr. Gorgi Yousr is the guarantor of the integrity of this study. 


\section{REFERENCES}

[1] Wiik AS, Høier-Madsen M, Forslid J, Charles P, Meyrowitsch J. Antinuclear antibodies: A contemporary nomenclature using HEp-2 cells. J Autoimmun 2010; 35: 276-90. http://dx.doi.org/10.1016/j.jaut.2010.06.019

[2] Meroni PL, Schur PH. ANA screening: an old test with new recommendations. Ann Rheum Dis 2010; 69: 1420-2. http://dx.doi.org/10.1136/ard.2009.127100

[3] Bizzaro N, Antico A, Platzgummer S, Tonutti E, Bassetti D, Presente $\mathrm{F}$, et al. Automated antinuclear immunofluorescence antibody screening: A comparative study of six computer-aided diagnostic systems. Autoimmun Rev 2014; 13: 292-8. http://dx.doi.org/10.1016/j.autrev.2013.10.015

[4] Copple SS, Giles SR, Jaskowski TD, Gardiner AE, Wilson AM, Hill HR. Screening for IgG antinuclear antibodies by Hep-2 indirect immunofluorescence antibody assays and the need for standardization. Am J Clin Pathol 2012; 137: 82530. http://dx.doi.org/10.1309/AJCPICNFG7UCES1S

[5] Hahm D, Anderer U. Establishment of Hep-2 cell preparation for automated analysis of ANA fluorescence pattern. Cytometry A 2006; 69: 178-81. http://dx.doi.org/10.1002/cyto.a.20223
[6]

Tonti S, Di Cataldo S, Bottino A, Ficarra E. An automated approach to the segmentation of Hep-2 cells for the indirect immunofluorescence ANA test. Comput Med Imaging Graph 2014.

http://dx.doi.org/10.1016/j.compmedimag.2014.12.005b

[7] Voigt J, Krause C, Rohwäder E, Saschenbrecker S, Hahn M, Danckwardt $M$, et al. Automated indirect immunofluorescence evaluation of antinuclear autoantibodies on Hep-2 cells. Clin Dev Immunol 2012; 2012: 651058. http://dx.doi.org/10.1155/2012/651058

[8] Egerer K, Roggenbuck D, Hienmann R, Weyer MG, Büttner $\mathrm{T}$, Radau B, et al. Automated evaluation of autoantibodies on human epithelial-2 cells as an approach to standardize cellbased immunofluorescence tests. Arthritis Res Ther 2010; 12: R40. http://dx.doi.org/10.1186/ar2949

[9] Hienmann R, Büttner T, Krieger T, Roggenbuck D, Sack U, Conrad K. Challenges of automated screening and differentiation of non-organ specific autoantibodies on Hep-2 cells. Autoimmun Rev 2009; 9: 17-22. http://dx.doi.org/10.1016/j.autrev.2009.02.033

(C) 2015 Gorgi et al.; Licensee Lifescience Global.

This is an open access article licensed under the terms of the Creative Commons Attribution Non-Commercial License (http://creativecommons.org/licenses/by-nc/3.0/) which permits unrestricted, non-commercial use, distribution and reproduction in any medium, provided the work is properly cited. 